\title{
PANDEMIC INFLUENZA PREPAREDNESS
}

\author{
Jan Fizzell \\ NSW Health Public Health Officer Training Program \\ NSW Department of Health \\ Paul K Armstrong \\ Biopreparedness Unit \\ NSW Department of Health

\section{James M Branley} \\ Nepean and Blue Mountains Pathology Service \\ Nepean Hospital
}

An influenza pandemic occurs when a new influenza virus emerges which is markedly different from recently circulating seasonal influenza viruses and is able to:

- infect people and cause disease (rather than, or in addition to, infecting other mammals or birds)

- spread readily from person to person

- spread widely among the population (because most people will have little or no immunity to it).

Because so many people may be affected, pandemics of influenza have historically had devastating consequences. It is essential that the community, businesses and all levels of government undertake planning and preparatory action beyond health and emergency planning to protect the community and minimise the impact of any pandemic.

The highly pathogenic avian influenza virus known as H5N1, currently circulating in domestic and wild bird populations around the world, is a public health concern because of its potential to transform into a pandemic strain. As long as the virus continues to circulate in birds and other animals, there will be opportunities for this virus to change and adapt to humans.

Recent planning developments in NSW and Australia include the Council of Australian Government's National Action Plan for Human Influenza Pandemic, the release of a revised Australian Health Management Plan for Pandemic Influenza and the development of the NSW Human Influenza Pandemic Plan.

\section{CONTAINMENT}

In the revised Australian Health Management Plan for Pandemic Influenza there is an increased emphasis on containing the influenza pandemic by preventing or minimising transmission and spread of the virus. Proposed

\footnotetext{
* Bug Breakfast is the name given to a monthly series of hour-long breakfast seminars on communicable diseases delivered by the NSW Department of Health's Division of Population Health.
}

strategies include border control measures, widespread adoption of good hygiene and infection control practices, isolation of the sick, quarantine of contacts, and the use of antivirals for those exposed. Successful containment should attenuate the burden on the health system and buy time for a vaccine to be developed and distributed.

Within NSW, early containment activities will include border control activities at Sydney airport and the early activation of pandemic influenza screening in Emergency Departments. Home quarantine will be imposed for contacts of cases and a whole-of-government planning group is considering arrangements for the implementation of this measure. The public health response will need to be extensive and efficient so that potential cases are identified quickly and their contacts traced and offered prophylaxis.

\section{THE CLINICIAN'S PERSPECTIVE}

For the clinician faced with an impending influenza pandemic, flexibility, good communication skills, the ability to deal with a high flow of information, and good observation and collaboration abilities (especially in the context of research) will be paramount. Early in the pandemic (at the containment stage) the clinician will need to deal with challenging diagnoses, control panic (in other staff and the public), treat presumptively, and negotiate competing system needs.

Later in the pandemic (at the maintenance stage), the clinician will need to adapt to the change in circumstances. This will involve reserving diagnostic capacity for atypical cases, reviewing treatment strategies, examining the best use of resources in a time of shortage, operating with a shortage or lack of trained staff due to sickness or social distancing measures, and coping with the personal prospect of illness. The clinician's use of continuous antiviral prophylaxis may also be causing side effects.

In the tail of the pandemic (during the recovery stage), there may be a second wave of infections. Those affected by the virus may require rehabilitation. Regular services disrupted by the pandemic will need to be reinstated. There is a strong likelihood that staff fatigue and mental health issues will come to the fore.

Throughout the pandemic there will need to be a breaking down of barriers between occupational groups, a willingness to work differently, and a strong commitment to infection control. By planning co-operatively prior to the pandemic occurring, some of the adverse effects may be mitigated. 